\title{
Synthesis, spectroscopic characterization and antibacterial activity of new series of Schiff base derived from 4-aminoantipyrine and 2-amino benzimidazole
}

\section{Abstract}

Background and objective: Compounds having imine or azomethine $(-\mathrm{C}=\mathrm{N}-)$ functional group are known as Schiff bases. Schiff bases compounds are found to be an active pharmacophore for the design and development of various bioactive lead compounds. In this study, several new Schiff base compounds have been synthesized and characterized.

Methods: Williamson ether synthesis process has been used to synthesize -alkyloxy and substituted benzyloxy of benzaldehyde. Differently substituted ether benzaldehydes used to react with 4-amino-1,5-dimethyl-2-phenyl-1,2-dihydro-3H-pyrazol-3-one in one hand and $1 \mathrm{H}$-benzo[d]imidazol-2-amine on the other hand to produce Schiff base compounds.

Results: Synthesized ether derivative compounds (3a-e) were converted to new series of Schiff bases (4a-e and $5 a-e)$ by condensation of equal molar amounts of compounds (3a-e) with different heterocyclic amines dissolved in absolute ethanol. All synthesized compounds were confirmed by (IR, ${ }^{1} \mathrm{H}-\mathrm{NMR}$, and ${ }^{13} \mathrm{CNMR}$ ) spectroscopy. All synthesized compounds were evaluated for antibacterial activities in vitro against Gram-positive and Gram-negative bacteria.

Conclusion: All compounds were purely synthesized, and all compounds were indicated growth inhibition against Escherichia coli, and Staphylococcus aureus, respectively with different inhibition zones staring from 13 to $33 \mathrm{~mm}$.

Keywords: Schiff base; Williamson ether synthesis; Biological activity; Azomethine.

\section{Introduction}

Heterocyclic compounds containing one or more hetero atoms having the range of applications in our life, such as used as pharmaceuticals, agrochemicals, and veterinary products. ${ }^{1,2}$ Schiff bases play a major role in their significant bio-activities and containing azomethine $(-\mathrm{N}=\mathrm{CH}-)$ as an active pharmacophore. ${ }^{3}$ Schiff bases considered as important starting materials to synthesis new drug design and intermediate in organic syntheses, or rubber additives. ${ }^{4}$ They have received much attention in the field of chemistry and biology due to their chemotherapeutic value. $^{5}$ Schiff bases are formed by the reaction between primary amine with an aldehyde or a ketone under specific conditions and used as amino protective groups in organic synthesis. ${ }^{6-8}$ Metal ions can form complex with Schiff bases as a ligand and liquid crystals in analytical, medicinal, and polymer chemistry. ${ }^{9,10}$ Based on the wide spectrum of biological profile of Schiff bases and heterocyclic compounds and their importance in pharmaceutical and biological fields, it was thought of interest to accommodate imidazole and pyrazolone moieties with alkyloxy and substituted benzyloxy group and azomethine bond in a single molecular framework to synthesis of new series of Schiff bases (Imines) of heterocyclic amine compounds for enhancing biological activity. This study aimed to synthesize several 4- hydroxyl benzaldehyde derivatives by a substitution reaction with different alkyl halide followed

${ }^{1}$ Department of Pharmaceutical Chemistry, College of Pharmacy, Hawler Medical University, Erbil, Iraq.

2 Department of Pharmacy, Aynda Private Technical Institute, Erbil, Iraq.

${ }^{3}$ Department of Clinical Biochemistry, College of Health Sciences, Hawler Medical University, Erbil, Iraq.

* Correspondence: sarbast.ahmed@hmu.edu.krd 
https://doi.org/10.15218/zjms.2019.026

by synthesis of series of new active pharmacophore Schiff base compounds (4a-e) and (5a-e) derived from 4-aminoantipyrine and 2minobenzimidazole with substituted benzaldehyde containing substituted benzyloxy and alkyloxy group in para position by a condensation reaction. The biological activity of the synthesized compounds studied on some of the gram-positive bacteria and gram-negative bacteria. Antipyrine (1,5-dimethyl-2phenylpyrazole-3-one) is a compound that possesses a pyrazolone moiety with a five-membered lactam ring containing two nitrogen and a ketone in the same molecule. Antipyrine and its 4-amino derivative (4-amino-1,5-dimethyl2-phenylpyrazole-3-one) have shown outstanding pharmacological properties such as anti-inflammatory, analgesic, antiviral, antipyretic, antirheumatic, and biological activity. ${ }^{11,12}$ Benzimidazole was the second heterocyclic compound used for organic synthesis and preparing a wide variety of substituted benzimidazoles and application of such compounds in the development of new chemotherapeutic agents are investigated. ${ }^{12}$ This study aimed to synthesize and characterize several new Schiff base compounds and to screen their antibacterial activity.

\section{Methods}

This experimental study was done at a College of Pharmacy, Hawler Medical University, from $20^{\text {th }}$ of February to $25^{\text {th }}$ of April 2018 starting from different derivatives of alkyl halides, phenol, and amines. Melting points were recorded using a Stuart scientific Melting Point (Table 1). Infrared spectra were recorded in the range 4000-600 cm-1 using a Shimadzu Scientific Instruments' IR, as $\mathrm{KBr}$ disc (Chemistry Department, College of Education, Salahadden University, Erbil. Samples were either thin films or powders. All absorptions are quoted in $\mathrm{cm}^{-1} .{ }^{1} \mathrm{H}-\mathrm{NMR}$ and ${ }^{13} \mathrm{C}$-NMR spectra were recorded on Brukeravance $(600 \mathrm{MHz})$ spectrometer. Chemical shifts are expressed in parts per million downfield from tetra-methylsilane as an internal standard. NMR spectra were recorded in solutions in deuterateddimethylsulfoxide (DMSO- $\mathrm{d}_{6}$ ).

Table 1: Some physical constants of synthesized compounds.

\begin{tabular}{|c|c|c|c|c|c|c|c|c|c|}
\hline Compds & $\begin{array}{c}\text { Chemical } \\
\text { formula }\end{array}$ & M.P & Yield & Color & Comp. & $\mathbf{R}-$ & M.P & $\begin{array}{c}\text { Yield } \\
\%\end{array}$ & color \\
\hline $4 a$ & $\mathrm{C}_{25} \mathrm{H}_{23} \mathrm{~N}_{3} \mathrm{O}_{2}$ & $146-148$ & 90 & $\begin{array}{l}\text { Light } \\
\text { yellow }\end{array}$ & $3 a$ & $\mathrm{C}_{6} \mathrm{H}_{4} \mathrm{CH}_{2} \mathrm{O}-$ & $63-65$ & 93 & white \\
\hline $4 b$ & $\mathrm{C}_{25} \mathrm{H}_{22} \mathrm{~N}_{4} \mathrm{O}_{4}$ & $167-170$ & 92 & yellow & $3 b$ & 3- $\mathrm{NO}_{2}-\mathrm{C}_{6} \mathrm{H}_{4} \mathrm{CH}_{2} \mathrm{O}-$ & $53-59$ & 87 & yellow \\
\hline $4 c$ & $\mathrm{C}_{22} \mathrm{H}_{25} \mathrm{~N}_{3} \mathrm{O}_{2}$ & $155-157$ & 80 & $\begin{array}{l}\text { Light } \\
\text { yellow }\end{array}$ & $3 c$ & $\mathrm{C}_{4} \mathrm{H}_{10} \mathrm{O}-$ & 253 & 85 & $\begin{array}{l}\text { Yellow } \\
\text { liquid }\end{array}$ \\
\hline $4 d$ & $\mathrm{C}_{26} \mathrm{H}_{22} \mathrm{BrN}_{3} \mathrm{O}_{3}$ & $176-178$ & 85 & Brown & $3 d$ & $4-\mathrm{Br}-\mathrm{C}_{6} \mathrm{H}_{5} \mathrm{COCH}_{2} \mathrm{O}-$ & $96-98$ & 90 & $\begin{array}{l}\text { Light } \\
\text { brown }\end{array}$ \\
\hline $4 e$ & $\mathrm{C}_{25} \mathrm{H}_{22} \mathrm{CIN}_{3} \mathrm{O}_{2}$ & $138-139$ & 83 & white & $3 e$ & $3-\mathrm{ClC}_{6} \mathrm{H}_{6} \mathrm{CH}_{2} \mathrm{O}-$ & $53-55$ & 85 & white \\
\hline $5 a$ & $\mathrm{C}_{21} \mathrm{H}_{17} \mathrm{~N}_{3} \mathrm{O}$ & $210-212$ & 85 & $\begin{array}{l}\text { Light } \\
\text { brown }\end{array}$ & & & & & \\
\hline $5 b$ & $\mathrm{C}_{21} \mathrm{H}_{16} \mathrm{~N}_{4} \mathrm{O}_{3}$ & $232-234$ & 85 & yellow & & & & & \\
\hline $5 c$ & $\mathrm{C}_{18} \mathrm{H}_{19} \mathrm{~N}_{3} \mathrm{O}$ & $239-241$ & 75 & $\begin{array}{l}\text { Light } \\
\text { yellow }\end{array}$ & & & & & \\
\hline $5 d$ & $\mathrm{C}_{22} \mathrm{H}_{16} \mathrm{BrN}_{3} \mathrm{O}_{2}$ & $243-245$ & 80 & brown & & & & & \\
\hline $5 e$ & $\mathrm{C}_{21} \mathrm{H}_{16} \mathrm{ClN}_{3} \mathrm{O}$ & $225-227$ & 75 & $\begin{array}{l}\text { Light } \\
\text { yellow }\end{array}$ & & & & & \\
\hline
\end{tabular}




\section{Results}

\section{Synthesis of 4-AlkoxyBenzaldehydes} $(3 a-e)^{4}$

A mixture of 4-hydroxybenzaldehyde (0.05mole) and $\mathrm{K}_{2} \mathrm{CO}_{3}$ (0.11 mole) was dissolved in $10 \mathrm{ml}$ of absolute ethanol and stirred at room temperature for 2 hours. 0.05 mole of alkyl halides were added to the mixture and heated under reflux for 7 hours. The mixture was powered into ice and filtered. Products were recrystallized from the suitable solvent (Table 2 and 3 ).

Synthesis of (phenylpyrazolonelidene) 4 -substitutedphenyl (4a-e ) ${ }^{4}$

4-aminoantipyrine(2.21 g, 0.01 mole) was dissolved in $10 \mathrm{ml}$ of absolute ethanol, appropriate aldehyde (0.01 mole) was added to the mixture. The reaction mixture was refluxed for 5 hours and then cooled. The products were precipitated and filtered off, recrystallized from absolute ethanol. All physical properties are listed in Table1 and 2.

Synthesis of benzimidazolidene - 2substituted phenyl ${ }^{4}$

2-aminobenzimidazole $(1.33 \mathrm{~g}, 0.01 \mathrm{~mole})$ was dissolved in $10 \mathrm{ml}$ of absolute ethanol; appropriate aldehyde (0.01 mole) was added to the mixture. The reaction mixture was refluxed for 5 hours and then cooled. The products were precipitated and filtered off and recrystallized from absolute ethanol. The physical properties are listed in Table 1 and 2.

Table 2: Infrared spectral data in $\mathrm{cm}^{-1}$ for synthesized compounds.

\begin{tabular}{|c|c|c|c|c|c|c|c|}
\hline Comp. & $C=0$ & NH & $\begin{array}{c}\mathrm{CH} \\
\mathrm{Ar}-\mathrm{H}\end{array}$ & $\begin{array}{l}\mathrm{CH}_{2} \\
\mathrm{R}-\mathrm{H}\end{array}$ & $\begin{array}{c}\text { C-H } \\
\text { Imine }\end{array}$ & $\begin{array}{c}\text { C-O } \\
\text { Ether }\end{array}$ & $\mathrm{N}=\mathrm{C}$ \\
\hline $4 a$ & 1637 & & 3034 & 2941 & 2883 & 1238 & 1602 \\
\hline $4 b$ & 1647 & & $\begin{array}{l}3099 \\
3076\end{array}$ & 2935 & 2887 & 1242 & 1608 \\
\hline $4 c$ & 1645 & & 3055 & $\begin{array}{l}2958 \\
2931 \\
2904\end{array}$ & 2872 & 1242 & 1597 \\
\hline $4 d$ & $\begin{array}{l}1654 \\
1701\end{array}$ & & 3061 & 2922 & 2850 & 1226 & 1654 \\
\hline $4 e$ & 1647 & & 370 & 2928 & 2872 & 1236 & 1608 \\
\hline $5 a$ & & 3350 & 3057 & 2829 & 2744 & 1261 & $\begin{array}{l}1687 \\
1600\end{array}$ \\
\hline $5 b$ & & 3300 & 3070 & 2952 & 2860 & 1247 & $\begin{array}{l}1685 \\
1604\end{array}$ \\
\hline $5 c$ & & 3300 & 3086 & 2958 & 2873 & 1246 & $\begin{array}{l}1685 \\
1604\end{array}$ \\
\hline $5 d$ & & 3350 & 3066 & 2972 & 2875 & 1217 & $\begin{array}{l}1693 \\
1598\end{array}$ \\
\hline $5 e$ & & 3230 & 3091 & 2879 & 2790 & 1220 & $\begin{array}{l}1685 \\
1602\end{array}$ \\
\hline
\end{tabular}


Table 3: ${ }^{1} \mathrm{H}-\mathrm{NMR}$ and ${ }^{13} \mathrm{C}-\mathrm{NMR}$ chemical shift assignment in $\mathrm{ppm}$ for synthesized compounds

Compounds

$4 b$

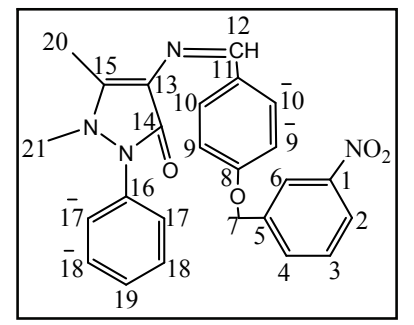

$4 \mathrm{c}$

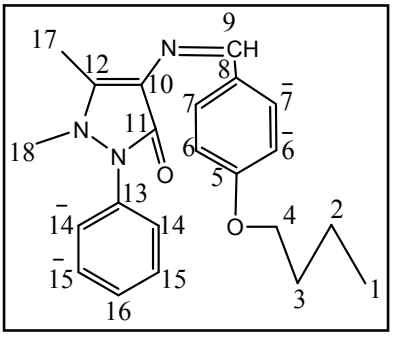

$4 d$

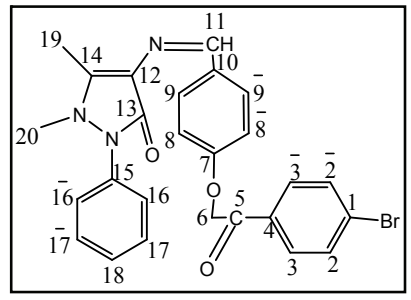

$4 \mathrm{e}$

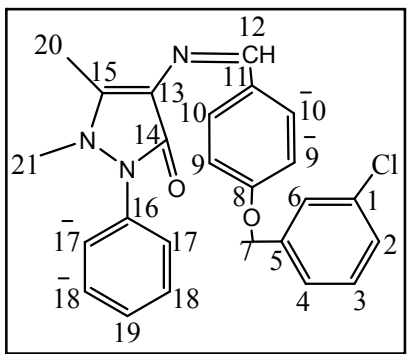

${ }^{1} \mathrm{H}$-NMR and ${ }^{13} \mathrm{C}-\mathrm{NMR}$ Chemical shifts in ppm

${ }^{1} \mathrm{H}$ NMR $\left(600 \mathrm{MHz}, \mathrm{DMSO}-d_{6}\right) \delta 9.52(\mathrm{~s}, 1 \mathrm{H}, \mathrm{CH}=\mathrm{CN})$,

7.34-7.77 (m, 14H, Ar-H), $5.17\left(\mathrm{~s}, 2 \mathrm{H}, \mathrm{CH}_{2}\right), 3.17\left(\mathrm{~s}, 3 \mathrm{H}, \mathrm{NCH}_{3}\right)$, 2.43(s, 3H, $\left.\mathrm{CH}_{3}\right),{ }^{13} \mathrm{C}$ NMR (151 MHz, DMSO) $\delta 160.58\left(\mathrm{C}_{10}\right)$, $160.29\left(\mathrm{C}_{12}\right), 154.69\left(\mathrm{C}_{6}\right), 152.34\left(\mathrm{C}_{13}\right), 137.27\left(\mathrm{C}_{4}\right), 135.16$

$\left.\left(\mathrm{C}_{14}\right), 131.01\left(\mathrm{C}_{9}\right), 129.57\left(\mathrm{C}_{8,8}\right)^{-}\right), 129.33\left(\mathrm{C}_{16,16}{ }^{-}\right), 128.93\left(\mathrm{C}_{2,2}\right)$, $128.38\left(\mathrm{C}_{1}\right), 128.18\left(\mathrm{C}_{3,3}{ }^{-}\right), 127.18\left(\mathrm{C}_{17}\right), 124.83\left(\mathrm{C}_{7,7}{ }^{-}\right), 117.41$

$\left(\mathrm{C}_{15,15^{\circ}}\right), 115.51\left(\mathrm{C}_{11}\right), 69.84\left(\mathrm{C}_{5}\right), 36.00\left(\mathrm{C}_{19}\right), 10.25\left(\mathrm{C}_{18}\right)$.

${ }^{1} \mathrm{H}$ NMR $\left(600 \mathrm{MHz}\right.$, DMSO- $\left.d_{6}\right) \delta 9.52(\mathrm{~s}, 1 \mathrm{H}, \mathrm{CH}=\mathrm{CN}), 7.13-$ $8.34(\mathrm{~m}, 13 \mathrm{H}, \mathrm{Ar}-\mathrm{H}), 5.34\left(\mathrm{~s}, 2 \mathrm{H}, \mathrm{CH}_{2}\right), 3.15\left(\mathrm{~s}, 3 \mathrm{H}, \mathrm{NCH}_{3}\right), 2.44$ (s, $3 \mathrm{H}, \mathrm{CH}_{3}$ ).

${ }^{13} \mathrm{C}$ NMR (151 MHz, DMSO) $\delta 160.25\left(\mathrm{C}_{12}\right), 160.14$ (C14), $154.56\left(\mathrm{C}_{8}\right), 148.34\left(\mathrm{C}_{15}\right), 139.70\left(\mathrm{C}_{1}\right), 135.14\left(\mathrm{C}_{5}\right), 134.64$

$\left(\mathrm{C}_{16}\right)$ 131.41( $\left.\mathrm{C}_{4}\right), 130.58\left(\mathrm{C}_{10,10^{-}}\right), 129.58\left(\mathrm{C}_{18,18^{-}}\right), 129.38$

$\left(\mathrm{C}_{3,11}\right), 127.21\left(\mathrm{C}_{19}\right), 124.86\left(\mathrm{C}_{2,6}\right), 123.28\left(\mathrm{C}_{17,17}\right), 117.41$

$\left(\mathrm{C}_{9,9}\right), 115.51\left(\mathrm{C}_{13}\right), 69.53\left(\mathrm{C}_{7}\right), 35.98\left(\mathrm{C}_{21}\right), 10.25\left(\mathrm{C}_{20}\right)$.

${ }^{1} \mathrm{H}$ NMR $\left(600 \mathrm{MHz}, \mathrm{DMSO}-d_{6}\right): \delta 9.52(\mathrm{~s}, 1 \mathrm{H}, \mathrm{CH}=\mathrm{CN})$, 7.0 - $7.8(\mathrm{~m}, 9 \mathrm{H}, \mathrm{Ar}-\mathrm{H}), 4.02\left(\mathrm{t}, 2 \mathrm{H}, \mathrm{CH}_{2}\right), 3.14\left(\mathrm{~s}, 3 \mathrm{H}, \mathrm{NCH}_{3}\right)$, $2.43\left(\mathrm{~s}, 3 \mathrm{H}, \mathrm{CH}_{3}\right), 1.74-1.68\left(\mathrm{~m}, 2 \mathrm{H}, \mathrm{CH}_{2}\right), 1.44\left(\mathrm{~h}, 2 \mathrm{H}, \mathrm{CH}_{2}\right)$, $0.94\left(\mathrm{t}, 3 \mathrm{H}, \mathrm{CH}_{3}\right)$.

${ }^{13} \mathrm{C}$ NMR (151 MHz, DMSO): $\delta$ 160.95) $\mathrm{C}_{9}$ ), 160.31) $\mathrm{C}_{11}$ ), 154.82) $\left.\left.\left.\left.\left.\mathrm{C}_{5}\right), 152.33\right) \mathrm{C}_{12}\right), 135.18\right) \mathrm{C}_{13}\right), 130$ )66. $\left.\mathrm{C}_{8}\right)$, 129.56) $\left.\left.\left.\left.\left.C_{7,7}{ }^{-}\right), 129.34\right) C_{15,15}{ }^{-}\right), 127\right) 15 . C_{16}\right), 124.83\left(C_{14,14}{ }^{-}\right)$, 117.30 $\left(\mathrm{C}_{6,6}{ }^{-}\right), 115.51\left(\mathrm{C}_{10}\right), 67.74\left(\mathrm{C}_{4}\right), 36.02\left(\mathrm{C}_{18}\right), 31.16\left(\mathrm{C}_{3}\right)$, $19.19\left(C_{2}\right), 14.16\left(C_{1}\right), 10.25\left(C_{17}\right)$.

${ }^{1} \mathrm{H}$ NMR $\left(600 \mathrm{MHz}, \mathrm{DMSO}-d_{6}\right): \delta 9.53(\mathrm{~s}, 1 \mathrm{H}, \mathrm{CH}=\mathrm{CN})$, 7.10- 7.77 (d, 13H, Ar-H), $5.19\left(\mathrm{~s}, 2 \mathrm{H}, \mathrm{CH}_{2}\right), 3.34(\mathrm{~s}, 3 \mathrm{H}$, $\left.\mathrm{NCH}_{3}\right), 3.15\left(\mathrm{~s}, 3 \mathrm{H}, \mathrm{CH}_{3}\right)$.

${ }^{13} \mathrm{C}$ NMR (151 MHz, DMSO): $\delta 191\left(\mathrm{C}_{5}\right), \delta 160.29\left(\mathrm{C}_{11}\right), 160.26$ $\left(\mathrm{C}_{13}\right), 154.62\left(\mathrm{C}_{7}\right), 152.38\left(\mathrm{C}_{14}\right), 139.88\left(\mathrm{C}_{15}\right), 135.15\left(\mathrm{C}_{4}\right)$, $133.60\left(\mathrm{C}_{2,2^{-}}\right), 131.20\left(\mathrm{C}_{3,3}{ }^{-}\right), 130.87\left(\mathrm{C}_{9,9}{ }^{-}\right), 129.58\left(\mathrm{C}_{17,17^{-}}\right)$, $129.35\left(\mathrm{C}_{1}\right), 128.30\left(\mathrm{C}_{10}\right), 127.83\left(\mathrm{C}_{18}\right), 127.19\left(\mathrm{C}_{8,8}{ }^{-}\right), 126.70$

$\left(\mathrm{C}_{16,16}\right), 115.52\left(\mathrm{C}_{12}\right), 68.88\left(\mathrm{C}_{6}\right), 35.99\left(\mathrm{C}_{20}\right), 10.25\left(\mathrm{C}_{19}\right)$.

${ }^{1} \mathrm{H}$ NMR $\left(600 \mathrm{MHz}\right.$, DMSO- $\left.d_{6}\right) \delta 9.50(\mathrm{~s}, 1 \mathrm{H}, \mathrm{CH}=\mathrm{CN})$, 7.1-7.8 (m, 13H, Ar-H), $5.54(\mathrm{~m}, 2 \mathrm{H}, \mathrm{CH} 2), 3.16\left(\mathrm{~s}, 3 \mathrm{H}, \mathrm{NCH}_{3}\right)$, $2.09\left(\mathrm{~s}, 3 \mathrm{H}, \mathrm{CH}_{3}\right)$.

${ }^{13} \mathrm{C}$ NMR (151 MHz, DMSO): $\delta 164.57\left(\mathrm{C}_{12}\right), 160.11\left(\mathrm{C}_{14}\right)$, $152.57\left(\mathrm{C}_{8}\right), 152.46\left(\mathrm{C}_{15}\right), 151.86\left(\mathrm{C}_{5}\right), 148.50\left(\mathrm{C}_{16}\right)$, $135.31\left(C_{1}\right) 132.64\left(C_{3}\right), 131.68\left(C_{10,10}{ }^{-}\right), 130.45\left(C_{18,18}\right)$, $129.56\left(\mathrm{C}_{2,6}\right), 126.30\left(\mathrm{C}_{11}\right), 124.84\left(\mathrm{C}_{4}\right), 123.05\left(\mathrm{C}_{19}\right)$, $122.95\left(\mathrm{C}_{9,9^{-}}\right), 119.74\left(\mathrm{C}_{17,17}\right), 116.19\left(\mathrm{C}_{13}\right), 56.85\left(\mathrm{C}_{7}\right)$, $35.96\left(\mathrm{C}_{21}\right), 10.21\left(\mathrm{C}_{20}\right)$. 


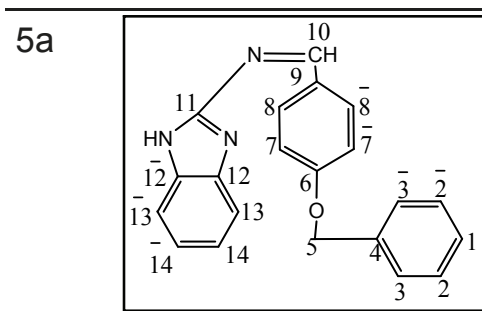

$5 b$

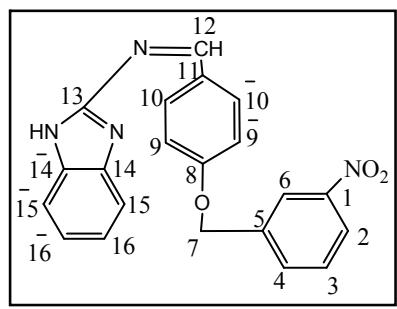

$5 c$

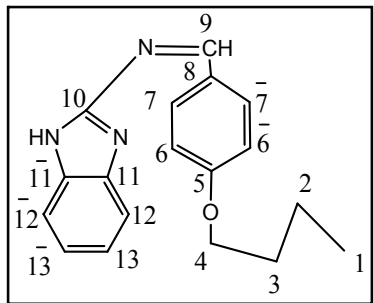

$5 d$

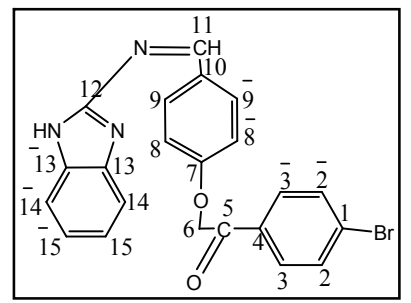

$5 e$

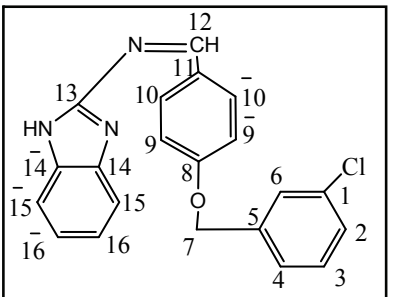

${ }^{1} \mathrm{H}$ NMR $\left(600 \mathrm{MHz}, \mathrm{DMSO}-d_{6}\right): \delta 9.88(\mathrm{~s}, 1 \mathrm{H}, \mathrm{CH}=\mathrm{CN})$, 7.22-7.89 (m, 13H, Ar-H), $5.24\left(\mathrm{~s}, 2 \mathrm{H}, \mathrm{CH}_{2}\right), 7.21(\mathrm{~s}, \mathrm{NH})$ ${ }^{13} \mathrm{C}$ NMR $\left(151 \mathrm{MHz}\right.$, DMSO) $\delta 191.87\left(\mathrm{C}_{6}\right), 163.79\left(\mathrm{C}_{10}\right)$, 163.59 $\left(\mathrm{C}_{11}\right)$ 136.49( $\left.\mathrm{C}_{4}\right), 132.26\left(\mathrm{C}_{12,12^{-}}\right)^{-}, 130.25\left(\mathrm{C}_{2,2}, 8,8^{-}\right)$, $128.98\left(\mathrm{C}_{1,9,3,3^{-}}{ }^{-}\right), 128.54\left(\mathrm{C}_{14,14}\right), 128.32\left(\mathrm{C}_{13,13^{-}}{ }^{-}\right), 115.75\left(\mathrm{C}_{7,7^{-}}\right)$, $70.13\left(\mathrm{C}_{5}\right)$.

${ }^{1} \mathrm{H}$ NMR $\left(600 \mathrm{MHz}, \mathrm{DMSO}-d_{6}\right): \delta 8.89(\mathrm{~s}, 1 \mathrm{H}, \mathrm{CH}=\mathrm{CN})$, 7.23-8,37 (m, 12H, Ar-H), 5.8 (s, 1H, NH), $5.42\left(\mathrm{~s}, 2 \mathrm{H}, \mathrm{CH}_{2}\right)$.

${ }^{13} \mathrm{C}$ NMR (151 MHz, DMSO): $\delta 184.10\left(\mathrm{C}_{8}\right), 165.44\left(\mathrm{C}_{12}\right)$, 148.36 $\left(\mathrm{C}_{13}\right), 140.20\left(\mathrm{C}_{1}\right), 134.95\left(\mathrm{C}_{5}\right), 134.71\left(\mathrm{C}_{14,14}{ }^{-}\right), 134.49\left(\mathrm{C}_{4}\right)$, $132.55\left(\mathrm{C}_{10,10^{-}}\right), 130.81\left(\mathrm{C}_{3}\right), 130.64\left(\mathrm{C}_{11}\right), 123.43\left(\mathrm{C}_{6}\right), 123.01$ $\left(C_{16,16}{ }^{-}\right), 122.75\left(C_{2}\right), 119.75\left(C_{15,15}\right), 115.97\left(C_{9,9}{ }^{-}\right), 68.76\left(C_{7}\right)$.

${ }^{1} \mathrm{H}$ NMR $\left(600 \mathrm{MHz}, \mathrm{DMSO}-d_{6}\right): \delta 9.80(\mathrm{~s}, 1 \mathrm{H}, \mathrm{CH}=\mathrm{CN})$, б 6.9- $7.9(\mathrm{~m}, 8 \mathrm{H}, \mathrm{Ar}-\mathrm{H}), 4.02\left(\mathrm{t}, 2 \mathrm{H}, \mathrm{CH}_{2}\right), 1.73-1.68(\mathrm{~m}, 2 \mathrm{H}$, $\left.\mathrm{CH}_{2}\right), 1.44$ (q, 2H, $\left.\mathrm{CH}_{2}\right), 0.93\left(\mathrm{t}, 3 \mathrm{H}, \mathrm{CH}_{3}\right), 6.7(\mathrm{~s}, \mathrm{NH})$. ${ }^{13} \mathrm{C}$ NMR (151 MHz, DMSO): $\delta$ 168.47(C $\left.\mathrm{C}_{9}\right), \delta 162.40\left(\mathrm{C}_{5}\right)$, $155.30\left(\mathrm{C}_{10}\right), 138.25\left(\mathrm{C}_{11,11}{ }^{-}\right), 131.70\left(\mathrm{C}_{7,7}\right), 125.04\left(\mathrm{C}_{8}\right)$, $120.04\left(\mathrm{C}_{13,13}{ }^{-}\right), 114.48\left(12,1^{-}\right), 111.87\left(\mathrm{C}_{6,6^{-}}{ }^{-}\right) 67.87\left(\mathrm{C}_{4}\right)$, $31.09\left(C_{3}\right), 19.16\left(C_{2}\right), 14.13\left(C_{1}\right)$.

${ }^{1} \mathrm{H}$ NMR $\left(600 \mathrm{MHz}, \mathrm{DMSO}-d_{6}\right) \delta 9.86(\mathrm{~s}, 1 \mathrm{H}, \mathrm{CH}=\mathrm{CN})$, 6.84- $7.90(\mathrm{~m}, 12 \mathrm{H}, \mathrm{Ar}-\mathrm{H}), 5.20\left(\mathrm{~s}, 1 \mathrm{H}, \mathrm{CH}_{2}\right) 7.10(\mathrm{~s}, \mathrm{NH})$. ${ }^{13} \mathrm{C}$ NMR $\left(151 \mathrm{MHz}\right.$, DMSO) $\delta 189\left(\mathrm{C}_{5}\right), \delta 168.47\left(\mathrm{C}_{7}\right)$, ठ 162.01( $\left.\mathrm{C}_{11}\right), 155.66\left(\mathrm{C}_{12}\right), 139.63\left(\mathrm{C}_{13,13}{ }^{-}\right), 133.60\left(\mathrm{C}_{4}\right)$, $131.80\left(\mathrm{C}_{2,2}{ }^{-}, 3,3^{-}\right), 130.90\left(\mathrm{C}_{9,9^{-}}\right), 128.37\left(\mathrm{C}_{10}\right), 128.88\left(\mathrm{C}_{1}\right)$, $126.75\left(\mathrm{C}_{15,15^{-}}\right), 119.45\left(\mathrm{C}_{14,14^{-}}\right), 115.04\left(\mathrm{C}_{8,8}{ }^{-}\right), 68.94\left(\mathrm{C}_{6}\right)$.

${ }^{1} \mathrm{H}$ NMR $\left(600 \mathrm{MHz}, \mathrm{DMSO}-d_{6}\right): \delta 9.87(\mathrm{~s}, 1 \mathrm{H}, \mathrm{CH}=\mathrm{CN})$, $6.25-8.23(\mathrm{~m}, 12 \mathrm{H}, \mathrm{Ar}-\mathrm{H}), 5.62\left(\mathrm{~s}, 2 \mathrm{H}, \mathrm{CH}_{2}\right) 6.17(\mathrm{~s}, \mathrm{NH})$. ${ }^{13} \mathrm{C}$ NMR (151 MHz, DMSO): $\delta 191.62\left(\mathrm{C}_{8}\right), 186.93\left(\mathrm{C}_{12}\right)$, $175.40\left(\mathrm{C}_{13}\right), 136.10\left(\mathrm{C}_{5}\right), 132.38\left(\mathrm{C}_{14,14^{-}}\right) 132.29\left(\mathrm{C}_{1}\right)$, $132.14\left(\mathrm{C}_{3}\right), 131.48\left(\mathrm{C}_{2,6}\right), 130.34\left(\mathrm{C}_{10,10}\right), 129.49\left(\mathrm{C}_{11}\right)$, $125.75\left(\mathrm{C}_{4}\right), 124.86\left(\mathrm{C}_{16,16^{-}}\right), 116.66\left(\mathrm{C}_{15,15}{ }^{-}\right), 115.49\left(\mathrm{C}_{9,9}{ }^{-}\right)$, $70.50\left(\mathrm{C}_{7}\right)$. 


\section{Biological Activity}

Mueller-Hinton agar medium was used to study the antibacterial activity of the synthesized compounds (2-5) against Escherichia coli and Staphylococcus aureus. $0.0512 \mathrm{~g}$ of each test compound was dissolved in $10 \mathrm{ml}$ of $20 \%$ of DMSO. Two different concentrations were prepared by using half of the prepared solution with $5 \mathrm{ml}$ of $20 \%$ DMSO with 256 and $512 \mu \mathrm{g} / \mathrm{ml}$ including another concentration to prepared by $5 \mathrm{ml}$ of $20 \%$ DMSO $128 \mu \mathrm{g} / \mathrm{ml}$. Sterile cork borer $(6 \mathrm{~mm})$ was used to prepare cups constructed in Petri plates, and $0.1 \mathrm{ml}$ of each tested compound was added separately into each well, and then bacterial plates were incubated at $37 \mathrm{C}$ in $24 \mathrm{hrs}$. The Zone of inhibition fashioned by each compound was measured in $\mathrm{mm}$ (Table 4).

\section{Discussion}

The first stage of this work involves the synthesis of ether derivatives forbenzaldehyde(3a-e) from $p$-hydroxyl benzaldehyde using the Williamson ether synthesis method. ${ }^{13}$ The reaction proceeded under reflux condition using potassium carbonate, and the nucleophilic substitution reactions were progressed smoothly, and products were obtained in very good yields, and high purity for the alkyloxy and substituted benzylxoybenzaldehyde. All synthesized compounds were studied by NMR and IR spectroscopy. IR spectra for all ether compounds showed the disappearance of $\mathrm{OH}$ band for p-hydroxyl benzaldehyde as a starting material that appeared at $(3400$ $\left.\mathrm{cm}^{-1}\right)$, whereas, new bands at $(1217-1352$ $\mathrm{cm}^{-1}$ ) were shown due to the new $\mathrm{C}-\mathrm{O}$

Table 4: Diameter of inhibited zones in millimeters as a measure of antibacterial activity of the synthesized compounds (4a-e and $5 \mathbf{a}-\mathbf{e}$ ) and some standard drugs.

\begin{tabular}{ccccc}
\hline \multirow{2}{*}{ Compounds } & \multicolumn{2}{c}{ Escherichia coli } & \multicolumn{2}{c}{ Staphylococcus aureus } \\
& $256 \mu \mathrm{g}$ & $512 \mu \mathrm{g}$ & $256 \mu \mathrm{g}$ & $512 \mu \mathrm{g}$ \\
\hline A & - & 32 & - & - \\
B & - & 24 & - & - \\
C & - & - & - & 28 \\
D & - & 14 & - & - \\
E & - & - & - & - \\
A & - & 18 & - & 13 \\
B & - & 20 & - & 33 \\
C & - & - & - & - \\
D & - & 22 & - & 19 \\
E & - & 25 & - & \\
Amikacin & $19-26$ & & $20-26$ & \\
Cefalotin & $15-21$ & & $29-37$ & \\
Tetracycline & $18-25$ & & $24-30$ & \\
Chloramphenicol & $21-27$ & & $19-26$ & \\
Nalidixic acid & $22-28$ & & -21 & \\
Vancomycin & - & & & \\
\hline
\end{tabular}


bond vibration indicated the formation of the desired ether compounds (vide infra) (Table 2). Ether derivatives of substituted benzaldehyde compounds (3a-e) have been synthesized successfully by using the previous conditions reacting with an appropriate amine in the presence of $\mathrm{K}_{2} \mathrm{CO}_{3}$ as a catalyst (Scheme 1). ${ }^{14}$ Substituted alkoxy and substituted benzyloxybenzaldehyde benzaldehyde were used as starting materials to react with 4-amino-antipyrineto prepare new series of Schiff bases (imines) using condensation method without the catalyst. All synthesized compounds were elucidated by IR and NMR spectroscopy. Synthesized compounds were washed with water to remove hydrophilic starting materials, and hexane has been used to remove trace amount of lipophilic materials. $\mathrm{IR},{ }^{1} \mathrm{H}$ - and ${ }^{13} \mathrm{C}-\mathrm{NMR}$ spectral data for synthesized compounds were consistent with expected structures. The Infrared spectra for the synthesized imines compounds showed the disappearance of the vibration frequency of $\mathrm{C}=\mathrm{O}$ groups at (1710) $\mathrm{cm}^{-1}$ of $\mathrm{C}=\mathrm{O}$ group for substituted of benzaldehyde and appearance of a new band for azomethines bond $(-\mathrm{N}=\mathrm{CH}-)$ in the region between (1597-1654) $\mathrm{Cm}^{-1}$ (Table 2). The bands at 3431 and 3400 $\mathrm{cm}^{-1}$ for 4-amino antipyrine are assigned to $\mathrm{N}-\mathrm{H}$ asymmetric and symmetric stretching vibrations disappeared in the IR spectrum of Schiff bases, the $\mathrm{C}=$ Ostretching vibration of pyrazolone ring observed at $1637-1654 \mathrm{~cm}^{-1}$ in the IR spectra of the synthesized compounds. Carbonyl bands are the most characteristics bands in Infrared spectra. The substituted benzene gives rise to $\mathrm{C}-\mathrm{H}$ stretching, $\mathrm{C}-\mathrm{H}$ out-ofplane bending and $\mathrm{C}-\mathrm{H}$ in-plane bendings. The bands around $3000-3100 \mathrm{~cm}^{-1}$ are assigned to- $\mathrm{H}$ stretching vibrations. The appearance of a singlet signal peak at $\delta$ (8-10) ppm showed azomethin protons $(-\mathrm{N}=\mathrm{CH})$ in ${ }^{1} \mathrm{HNMR}$ and significant peaks were distinguishable for substituted benzaldehyde for all synthesized compounds. Compound $\mathbf{4 a}$ is selected as a representative of their series for the study of ${ }^{1} \mathrm{H}-\mathrm{NMR}$, singlet signal atठ (9.5) ppm with a single signal for $\left(\mathrm{CH}_{2} \mathrm{O}\right)$ group at $\delta 5.2 \mathrm{ppm}$ indicates the presence of

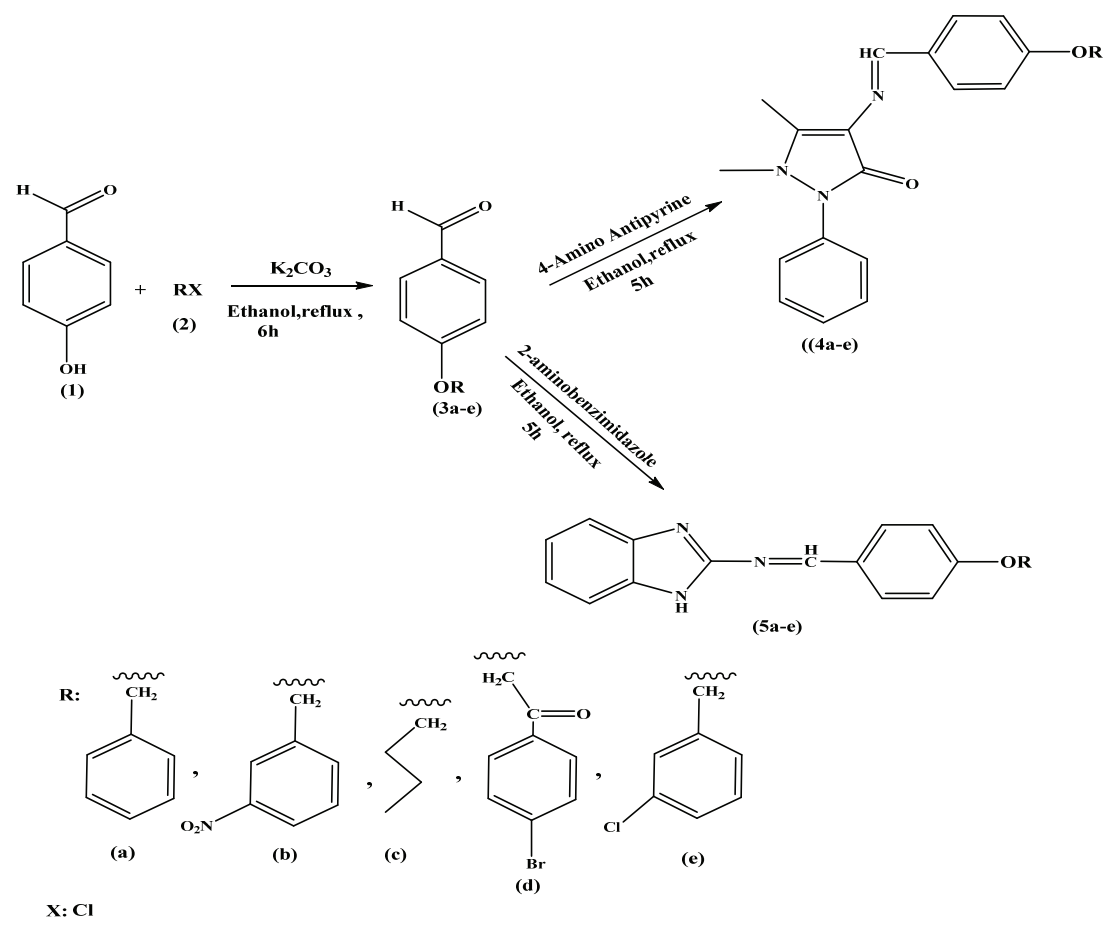

Scheme 1: Schiff base synthetic compound's pathway. 
benzyloxy $\left(\mathrm{C}_{6} \mathrm{H}_{5} \mathrm{CH}_{2} \mathrm{O}-\right)$ group in the methyl groups $\left(\mathrm{CH}_{3}\right)$ for pyrazolone moiety structure of a new compound and the multiplet signals from $\delta 7.2-7.7 \mathrm{ppm}$ for 14 protons of two phenyl rings. The two observed at $\delta 2.44\left(\mathrm{CH}_{3}\right)$ ppm and $\delta 3.1$ $\left(\mathrm{NCH}_{3}\right)$ (Figure 1 and Table 3).
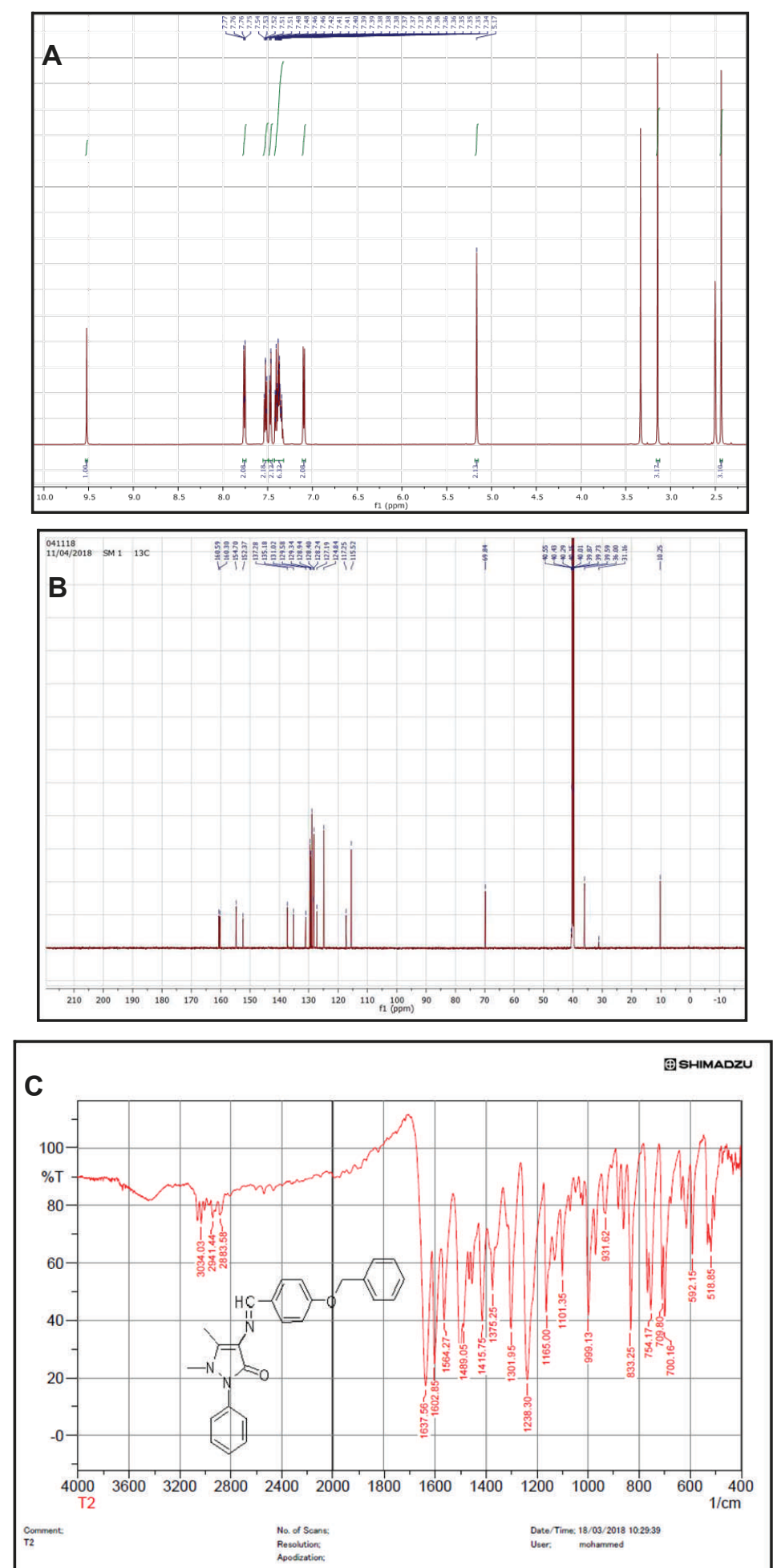

Figure 1: $(A){ }^{1} \mathrm{H}-\mathrm{NMR},(\mathrm{B}){ }^{13} \mathrm{C}-\mathrm{NMR}$, and $(\mathrm{C})$ IR spectra of compound $4 \mathrm{a}$. 
Further verification for the formation of new Schiff bases was attained from ${ }^{13} \mathrm{C}-\mathrm{NMR}$ spectra (Table 3); with the signal at(68-70) ppm due to the carbon atom that attached to oxygen atom $\mathrm{CH}_{2} \mathrm{O}$ group confirms the substitution reaction on hydroxyl group. The depicted signals in the aromatic region for the different type of carbons at different chemical shifts were inconvenient with a number of carbon atom in each compound with the observation of lines at $\delta$ (160-186) ppm related to azomethines carbon $(-\mathrm{N}=\mathrm{CH})$ (vide supra). The spectra of compounds (4a-e ) also revealed several absorption bands related to the reacted 4-aminoantipyrineat $\delta 10 \mathrm{ppm}, 35 \mathrm{ppm}$ and $\delta 152-160 \mathrm{ppm}$ attributed to the two methyl group- and carbonyl group $(\mathrm{C}=\mathrm{O})$ of pyrazolone moiety (Table 3 ). The aromatic carbon for compounds(5a-e) showed different type of carbons in different chemical shift at $\delta 164-115 \mathrm{ppm},{ }^{13} \mathrm{C}-\mathrm{NMR}$ data for compound $5 \mathrm{c}$ provided confirmatory evidence for substitution reaction on hydroxyl group exhibited four lines at 14, 19,31,68 ppm, respectively for butoxy group $\mathrm{CH}_{3} \mathrm{CH}_{2} \mathrm{CH}_{2} \mathrm{CH}_{2} \mathrm{O}$ - supported the formation of Schiff base via azomethines carbon $(-\mathrm{N}=\mathrm{CH})$ resonance at $168 \mathrm{ppm}$ (Table 3 and Figure 2).

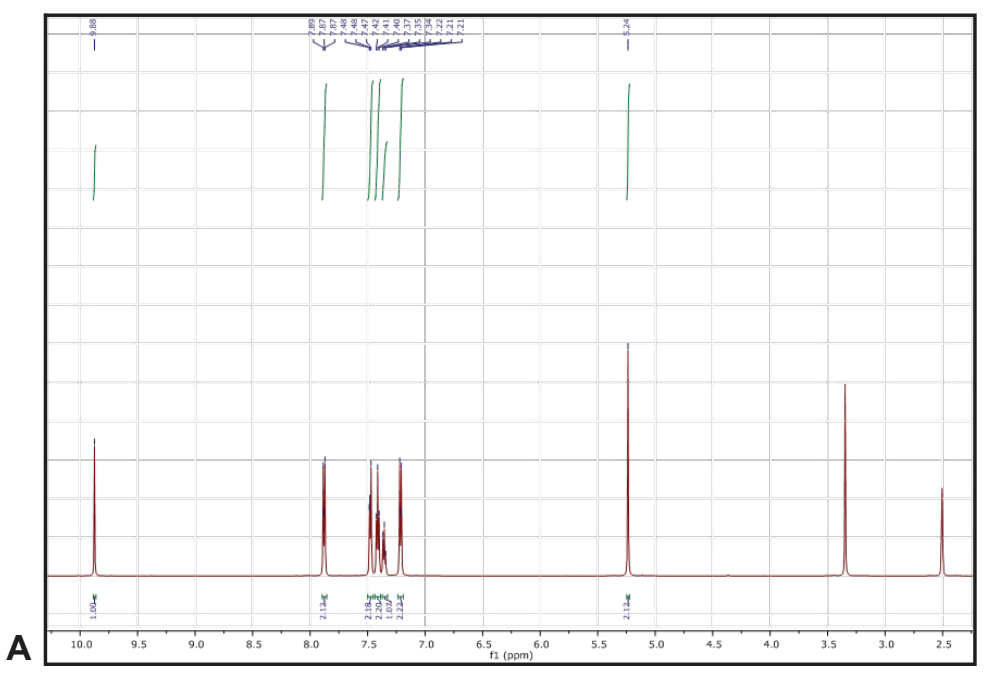

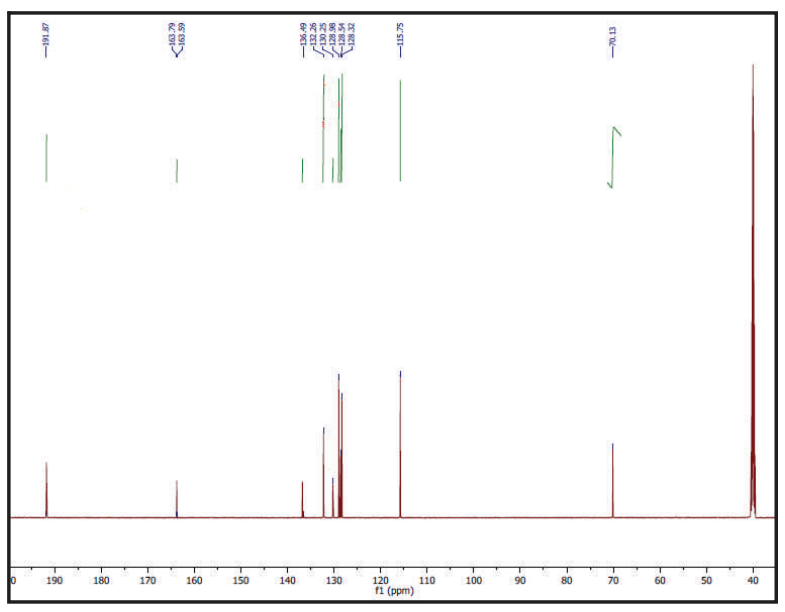

B

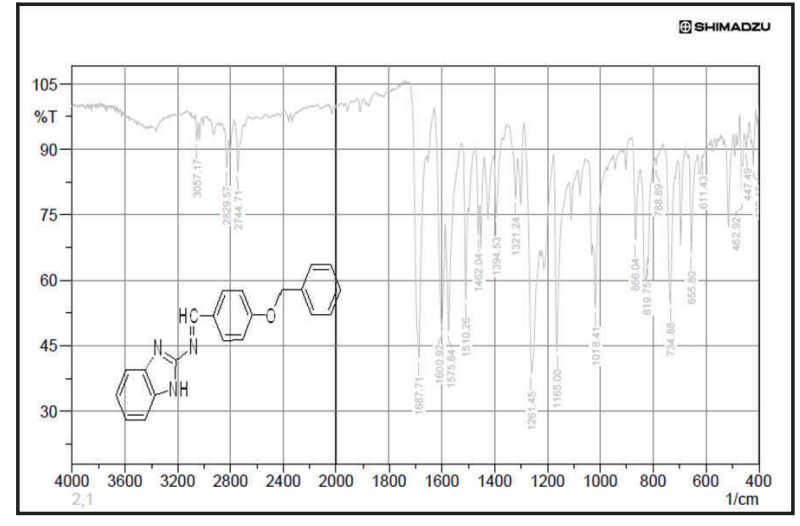

C

Figure 2: (A) ${ }^{1} \mathrm{H}-\mathrm{NMR},(\mathrm{B}){ }^{13} \mathrm{C}-\mathrm{NMR}$, and (C) IRspectra of compound $\mathbf{5 a}$. 


\section{Biological activity}

The biological activity of the present compounds in terms of the anti-bacterial property was analyzed against two well known pathogenic gram-negative and gram -positive organisms such as Escherichia coli, and Staphylococcus aureus, respectively. The activity was tested after the dissolution of all the compounds 4, 5 (a-e) in DMSO, which was used as a negative control in the experiment. The results of the bacterial growth inhibition are shown in Table 4 (vide supra) along with the corresponding positive and negative controls. All synthesized compounds except $\mathbf{4 c}, \mathbf{4 e}$, and $\mathbf{5 c}$ showed significant growth inhibition (14-32 $\mathrm{mm})$ against Escherichia coli, indicating that the experimental set and procedures are appropriate for the test. The most active compound against escherichia coli was compound $4 \mathrm{a}$ with $32 \mathrm{~mm}$ zone. Whereas, compound $4 \mathrm{~b}, 4 \mathrm{e}$, and $5 \mathrm{c}$ indicated antidistinguishable inhibition zone against Staphylococcus aureus organism with an inhibition zone of $13-33 \mathrm{~mm}$. With this exception, compounds $5 \mathrm{~b}$, and $\mathbf{5 e}$ showed inhibition zone against both Escherichia coli, and Staphylococcus aureus with inhibition zones of 20, 25, and 13, $19 \mathrm{~mm}$, respectively (Table 4 ).

\section{Conclusion}

In the present work, several substituted Schiff bases have been synthesized via using substituted 4-alkyloxy and substituted benzyloxybenzaldehyde aldehydes that prepared by hydroxyl benzaldehyde with different alkyl and substituted benzyl halide. The 4-alkyloxy andaldehydes were used as starting material to react with the different substituted amine to produce successfully several Schiff base compounds. 4-amino-1,5-dimethyl-2-phenyl -1,2-dihydro-3H-pyrazol-3-one was used to react with substituted 4-alkyloxy and aryloxyaldehydes to produce pure Schiff base compounds (4a-e). 1H-benzo[d] imidazol-2-amine was also used to react with substituted 4-alkyloxy substituted beznyloxy benzaldehyde in high purity without using chromatography techniques, and using sometimes hexane to purify products. All synthesized compounds were screened in biological activity indicated significant growth inhibition against Escherichia coli, and Staphylococcus aureus, respectively with different inhibition zones staring from 13 to $33 \mathrm{~mm}$.

\section{Competing interests}

The authors declare that they have no competing interests.

\section{References}

1. Azab ME, Rizk SA, Amr AE-GE. Synthesis of some novel heterocyclic and schiff base derivatives as antimicrobial agents. Molecules 2015; 20(10):18201-18.

2. Brzezinska-Rodak M, Peczynska-Czoch W. Biodegradation of $\mathrm{N}$-heterocyclic compound. Biotechnologia 2000; 1:102-16.

3. Kumar J, Rai A, Raj V. A comprehensive review on the pharmacological activity of schiff base containing derivatives. Org Med Chem J 2017; 1:555-64.

4. Brodowska K, Lodyga-Chruscinska E. Schiff bases-interesting range of applications in various fields of science. Chem Inform 2015; 46(11).

5. Ashraf MA, Mahmood K, Wajid A, Maah MJ, Yusoff I. Synthesis, characterization and biological activity of Schiff bases. IPCBEE 2011; 10:1-7.

6. Qin W, Long S, Panunzio M, Biondi S. Schiff bases: A short survey on an evergreen chemistry tool. Molecules 2013; 18(10):12264-89.

7. MOHAMED GG, Omar MM, Hindy AM. Metal complexes of Schiff bases: preparation, characterization, and biological activity. Turkish Journal of Chemistry 2006; 30(3):361-82.

8. Deshmukh P, Kumar P, Kankoriya A, Halve AK, Dixit R. 4-Aminoantipyrine: a significant tool for the synthesis of biologically active Schiff bases and metal complexes. Int $\mathrm{J}$ Pharm Sci Rev Res 2015; 34(1):162-70.

9. Prakash A, Adhikari D. Application of Schiff bases and their metal complexes-A Review. Int J Chem Tech Res 2011; 3(4):1891-6.

10. Ibrahim MN, Sharif SE. Synthesis, characterization and use of Schiff bases as fluorimetric analytical reagents. Journal of Chemistry 2007; 4(4):531-5.

11. Ali P, Meshram J, Sheikh J, Tiwari V, Dongre R, Hadda TB. Predictions and correlations of structure activity relationship of some aminoantipyrine derivatives on the basis of theoretical and experimental ground. Medicinal Chemistry Research 2012; 21(2):157-64. 
Synthesis, spectroscopic characterization .......

Zanco J. Med. Sci., Vol. 23, No. (2), August, 2019

https://doi.org/10.15218/zjms.2019.026

12. Alam MS, Lee D-U, Bari ML. Antibacterial and cytotoxic activities of Schiff base analogues of 4-aminoantipyrine. Journal of the Korean Society for Applied Biological Chemistry 2014; 57(5):6139.

13. Li JJ. Williamson ether synthesis. Name Reactions: A Collection of Detailed Mechanisms and Synthetic Applications Fifth Edition. Cham: Springer International Publishing; 2014. p. 628.

14. Ahmad M, Siddiqui HL, Ahmad S, Irfan Ashiq M, Tizzard GJ. Methyl 4-acetoxy-2-methyl-2H-1, 2-benzothiazine-3-carboxylate 1, 1-dioxide. Acta Crystallographica Section E: Structure Reports Online 2008; 64(3):0594. 